\title{
CARACTERIZACIÓN DE LA COMPOSICIÓN CORPORAL DE LOS JUGADORES DE LA SELECCIÓN BOYACÁ MASCULINA DE FÚTBOL SALA FIFA.
}

\author{
Uscategui. Juan ${ }^{1}$, Caro. William², Castro. Hugo ${ }^{3}$
}

Juan Manuel Uscategui Orejuela 1: Profesor Fundación Universitaria Juan de Castellanos, Especialista en Dirección y Gestión Deportiva, Entrenador selección Boyacá de Futbol Sala FIFA, juscateguil@jdc.edu.co

William Fernando Caro Cely 2: Magister en Pedagogía de la Cultura Física, Profesor Fundación Universitaria Juan de Castellanos.wcaro@jdc.edu.co

Hugo Cesar Castro Lara 3: Aspirante a Magister en pedagogía de la Cultura Física, Entrenador de Natación. hugocesar27@hotmail.com.

\section{RESUMEN}

El objetivo del presente estudio fue el de caracterización de la composición corporal de los jugadores de la selección Boyacá masculina de fútbol sala FIFA, se trabajó con un total de 14 deportistas, con una edad promedio de 25,1 años, un peso de $69,7 \mathrm{~kg}$ y una estatura de $171,5 \mathrm{~cm}$. El equipo presentó un somatotipo mesomorfo balanceado con predominio del componente muscular sobre el graso. El estudio demuestra que los jugadores se encuentran en un somatotipo óptimo para la competencia, además aporta datos biotipológicos actualizados de referencia para la población competitiva del Futbol Sala FIFA.

PALABRAS CLAVES: Antropometría, Composición Corporal, Futbol sala FIFA.

\begin{abstract}
The objective of the present study was to characterize the body composition of the players of the soccer team of the FIFA room, worked with a total of 14 athletes, with an average age of 25.1 years, a weight of $69,7 \mathrm{~kg}$ and a height of $171.5 \mathrm{~cm}$. The equipment is presented in a balanced mesomorph somatotype with predominance of the muscular component on the fat. The study demonstrates that players are in an optimal somatotype for the competition, in addition to the updated biotipological data of reference for the competitive population of the Futbol Sala FIFA.
\end{abstract}

KEYWORDS: Anthropometry, Body Composition, FIFA Futsal. 


\section{INTRODUCCION}

El futbol sala en Boyacense ha tomado posicionamiento a nivel nacional desde la creación de la Liga Argos Futsal en 2011, teniendo el departamento de BOYACÁ en este trasegar un subtítulo, tres clasificaciones a cuartos de final, dos convocados a selección nacional en la categoría sub 20, mayores y la reciente clasificación a los juegos nacionales 2015. Este auge denota que los deportistas del departamento poseen unas características especiales desde el enfoque biopsicosocial que lo diferencian de los jugadores de otras regiones.

El análisis de las características individuales y grupales de los jugadores son determinantes en la consecución de éxitos deportivos y de los modelos tácticos, por tal motivo la composición corporal juega un papel relevante en la forma física de los deportistas lo cual ha desencadenado una serie de estudios sobre el tema (De Ross, 2004).

El futbol sala a nivel mundial se ha posicionado fuertemente, teniendo en cuenta que depende de la FIFA y es una modalidad del fútbol. Por lo tanto a nivel suramericano sus más fuertes exponentes son Brasil y Argentina, estando Colombia con su liga en una cuarta posición y aportas de realizar el mundial en el 2016, a pesar de todo este realce de la disciplina no se generan amplios escritos teóricos de tinte científicos que caracterices los diferentes tópicos del futsal.

Por lo tanto, el interés de esta investigación es generar un modelo característico de los deportistas boyacenses, creado una base sólida para la formación de los futuros deportistas del departamento y generar estrategias de desarrollo deportivo a través de la ciencia.

Por lo tanto, se establece que el futsal es un deporte que posee características de duración y alta intensidad (Barbero, 2006) lo cual demanda poseer una adecuada composición corporal desde lo morfofuncional y teniendo en cuenta la función que cada jugador cumple dentro de un esquema.

\section{MARCO METODOLOGICO}

\section{Tipo de estudio}

La investigación que se plantea es de tipo descriptiva, con un enfoque cuantitativo ya que se caracteriza la población objeto de estudio teniendo en cuenta la información recolectada se caracterizara la población desde la composición corporal.

\section{Población y muestra}

La población está determinada por jugadores del género masculino de diferentes ciudades del departamento, pertenecientes a la selección Boyacá de futbol sala FIFA, que competirán en los juegos deportivos nacionales 2015.

La muestra está conformada por 14 jugadores de futbol sal FIFA, esta muestra se elige por conveniencia ya que son los jugadores que replantaran al departamento.

\section{Métodos y técnicas de recolección}

El método de recolección de la información es indirecto ya que la información que se obtiene para cada una de las muestras, es tomada del deportistas de forma tangible por medio de distintas medicines antropométricas, y la técnica utilizada para la recolección de información es no invasiva debido a que todas las médicos son realizadas en superficies corporales

\section{Variables antropométricas}

Masa corporal: cantidad de materia presente en un cuerpo humano.

Talla: Es la distancia máxima entre dos puntos del cuerpo humano (Lohman, Roche y Martorell, 1988) "Talla de pie, Talla sentado, Envergadura"

Pliegues cutáneos: espesor de una doble capa de piel y del tejido adiposo subcutáneo. "tríceps, bíceps, escapular, iliaco, abdominal, mulos medial, gemelo"

Perímetros: medición de la longitud o amplitud delimitada por dos puntos óseos (Behnke, A, 1996). "brazo relajado, brazo contraído, antebrazo, muñeca, tórax relajado, tórax expandido, cintura, cadera, muslo medial, gemelo, tobillo" 
Diámetros: medie las distancias entre los puntos óseos. "Estiloideo, biepicondilar humeral, biepicondilar femoral"

\begin{tabular}{|c|c|c|c|}
\hline IMPLEMENTO & MARCA & FUNCIÓN & $\begin{array}{l}\text { UNIDAD } \\
\text { MEDIDA }\end{array}$ \\
\hline Bascula o peso & $\begin{array}{c}\text { Sport } \\
\text { Fitness }\end{array}$ & $\begin{array}{l}\text { Medición de } \\
\text { la masa } \\
\text { corporal total }\end{array}$ & Kilogramos \\
\hline Tallímetro & Medical & $\begin{array}{c}\text { Medición de } \\
\text { la altura del } \\
\text { vértex y } \\
\text { estatura } \\
\text { sentado }\end{array}$ & Centímetros \\
\hline Cinta métrica & Polar & $\begin{array}{l}\text { Medición dos } \\
\text { perímetros y } \\
\text { puntos } \\
\text { medios }\end{array}$ & Centímetros \\
\hline $\begin{array}{l}\text { Paquimetro o } \\
\text { compas de } \\
\text { pequeños } \\
\text { diámetros }\end{array}$ & Discover & $\begin{array}{l}\text { Medición de } \\
\text { diámetros } \\
\text { óseos }\end{array}$ & Centímetros \\
\hline $\begin{array}{c}\text { Plicometro o } \\
\text { compas de } \\
\text { pliegues }\end{array}$ & Polar & $\begin{array}{c}\text { Medición del } \\
\text { espesor del } \\
\text { tejido } \\
\text { adiposo }\end{array}$ & Centímetros \\
\hline
\end{tabular}

TABLA N¹. Instrumentos de Recolección de Información.

\section{RESULTADOS:}

Interpretación de los resultados;

\begin{tabular}{|c|c|c|c|c|c|c|c|c|c|}
\hline UPO & Hassico & & 100 & & & & & & $x$ \\
\hline & NEDOO & 5,3 & 70,5 & 171,8 & 13,9 & 100 & & 48,8 & 941] \\
\hline & DESESET. & 4 & , & 0,1 & & o & & {$[, \mathrm{T}$} & \\
\hline \multirow{2}{*}{ R.5 } & WLW & 249 & 690 & $1 / 1 / 0$ & 6,4 & 900 & & 4,1 & \\
\hline & DESESET. & 3,2 & 1,3 & 60 & 1, & 1.8 & 4, & 22. & \\
\hline \multirow{2}{*}{ BOS } & NEDOO & 5, & 68,1 & 170,0 & 13,5 & 9,3 & 14,4 & 4,1 & \\
\hline & DESVEST. & $\sqrt{1}$ & 7,4 & 5,3 & 40 & 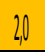 & $4^{47}$ & 2,5 & 18012 \\
\hline & NEDO & 25,0 & 71,3 & 172,7 & 33,9 & 9,2 & $8,10^{\circ}$ & 49,4 & .0991 \\
\hline & DSSEST. & 2,1 & 5,5 & 4,3 & & 2,0 & & 2,5 & 1.991. \\
\hline
\end{tabular}

TABLA N`2. Características generales por posición de juego

Se observa que en el los componentes de edad, peso, talla, IMC, \% óseo y \% muscular no se encuentran diferencias significativas por posición de juego, exceptuando el \% graso en donde los porteros evidencian valores elevados respecto al grupo; En las coordenadas del somatotipo $X$ los porteros tiene el valor más alto y los cierres el valor más bajo, lo contrario ocurre con $Y$ teniendo los pívot el valor más alto y el más bajo los cierres.

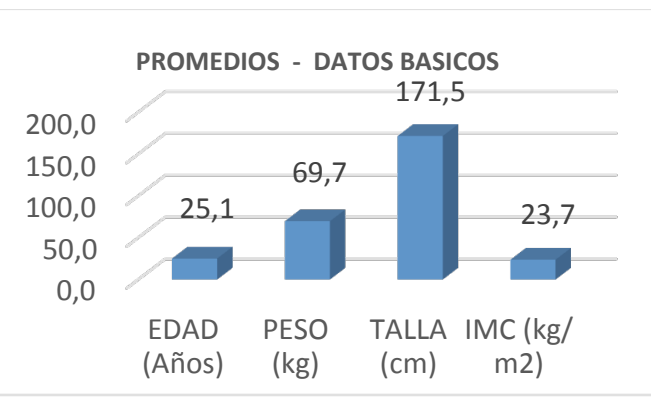

FIGURA №1. Componentes básicos (promedios, edad, peso, talla, IMC) de los deportistas evaluados.

En los cuatro indicadores básicos se puede caracterizar que los jugadores de fútbol sala FIFA, de la selección Boyacá tiene un promedio de edad 25,1 años lo cual se considera un grupo joven para un certámenes nacional, un peso de 69,7 y una estatura de 171,5 teniendo una relación positiva entre estos dos componentes; La composición corporal es de 23,7 encontrándose en el percentil 50 (Alba 2005).

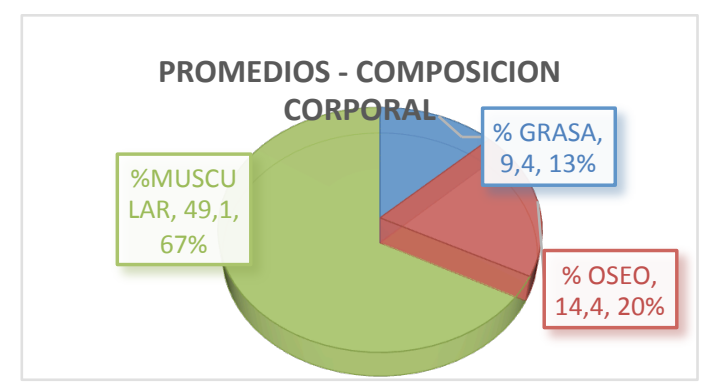

FIGURA N². Componentes corporales (grasa, musculo, óseo) de los deportistas estudiados.

Teniendo en cuenta los teres tejidos escogidos para la investigación, la característica del grupo es: Tejido óseo se encuentra 14,4 con una equivalencia de $20 \%$, Tejido muscular tiene un valor de 49,1 relacionándolo con el $67 \%$, por lo tanto la relación entre el componente óseo y 
muscular es adecuada; "En cuanto mayor es la proporción de masa muscular en relación al esqueleto, con mayor potencia se podrá trasladar el cuerpo en el espacio" (Alba 2005); El tejido graso tiene un valor de 9,4 equivalente al $13 \%$ considerándose adecuado para el deporte (Alba 2005).

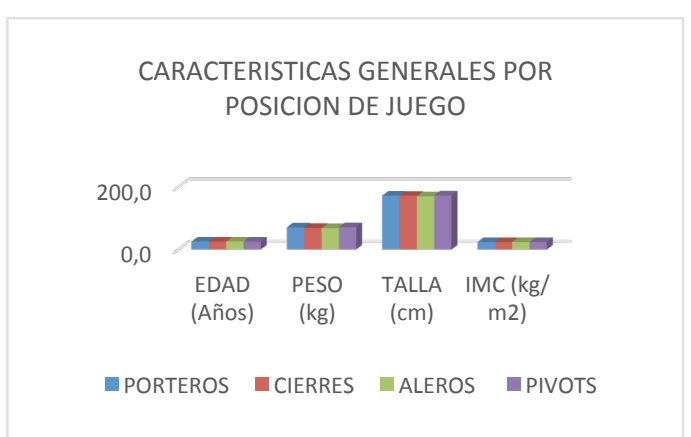

FIGURA N³. Componentes generales por posición de juego (edad, peso, talla, IMC) de los deportistas estudiados.

La figura 4 muestra que en cada uno de los componentes por posición de juego no se observan diferencias significativas, lo cual lleva a analizar que es un grupo homogéneo en sus características generales.

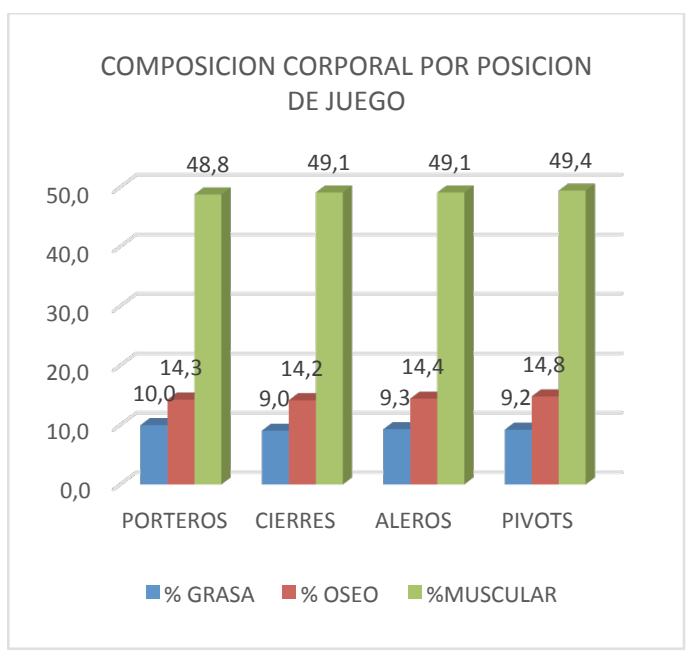

FIGURA $\mathbf{N}^{\circ} 4$. Componentes generales por posición de juego (grasa, musculo, óseo) de los deportistas estudiados.

Dentro de los factores de la composición corporal no se observan relevantes entre los cierres, aleros y pívot; Pero si en los porteros en su $\%$ de grasa 10,0, considerándose un valor por encima del ideal.

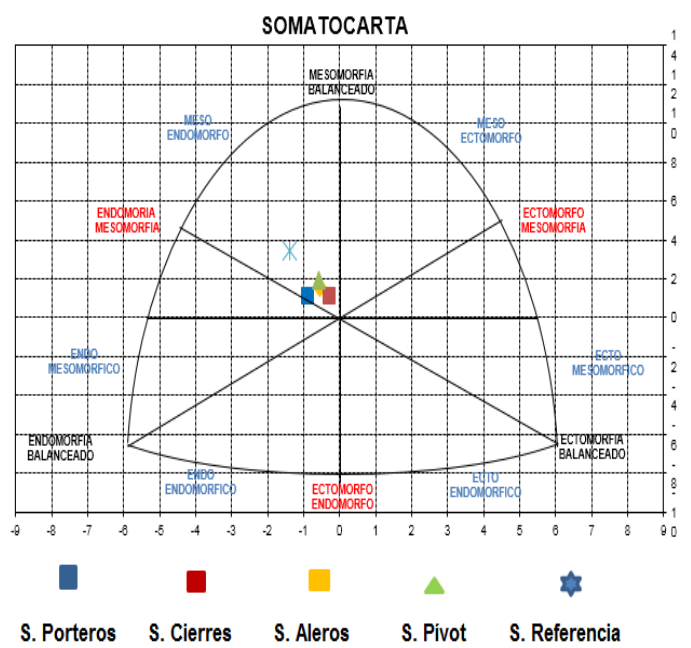

FIGURA ${ }^{\circ} 5$. Somatocarta del grupo estudiado $y$ de un grupo de referencia (jugadores de futbol sala FIFA selección Boyacá)

La evaluación del somatotipo (Figura 5), no evidenció diferencias en relación a la posición de juego ya que la distancia de dispersión del somatotipo medio globales no superó los dos puntos, Hay que destacar que el somatotipo que predomina en el grupo es mesomorfo balanceado, esto es, un predominio del compartimento muscular sobre los componentes grasos y de linealidad corporal, existiendo un equilibrio entre estos dos últimos.

\section{DISCUSIÓN:}

A pesar de los escasos trabajos realizados en jugadores de futbol sala FIFA, la estimación de los diferentes tejidos de la composición corporal se busca cuantificar cada uno de estos componentes, por medio de mediciones no invasivas y precisas que les permitan a los entrenadores caracterizar y monitorizar la evolución del deportista a lo largo de la temporada.

Por tal motivo este estudio ha permitido iniciar el camino a la construcción del modelo característico del jugador boyacenses, buscando precisar la primera parte de los componentes del desarrollo biopsicosociales. 
El estudio mostro que en la medidas básicas los jugador pertenecientes a la región que se encuentra ubicada en altitudes intermedias como es la ciudad de Tunja "2800msnm" poseen estaturas promedio de $171,1 \mathrm{~m}$ con pesos de $69,7 \mathrm{~kg}$ estando $3 \mathrm{~kg}$ por debajo del adecuado, pero con una masa activa de $67,9 \mathrm{~kg}$ lo cual concluye que el cociente de altura peso es de $41,6 \%$.

La composición corporal de jugadores esboza niveles adecuados teniendo en cuenta que el tejido muscular es mayor que el óseo, lo cual produce un aumento en la potencia (Sinaki y Offord 1988; Alba 2005) característica indispensable en el fútbol sala; cabe resaltar que el porcentaje de grada es adecuado con relación a la demanda energética que se requiere

Dentro del desarrollo por posición de juego en los componentes generales los pívots poseen mayor estatura que las restantes posiciones, teniendo clara la importancia de la estatura en las acciones tácticas de espaldas al arco. De igual manera se encuentra en la composición corporal por posición, un aumento en el tejido graso de los porteros, resultado dado por la ejercitación y la intermitencia dentro del juego.

La determinación del somatotipo de los jugadores participantes en este estudio, ha mostrado un predominio claro del componente mesomórfico sobre los dos componentes restantes; en relación con investigaciones biotípicas realizadas en este deporte, han demostrado que los jugadores de futbol sala, suelen tener un buen desarrollo muscular, (Lentini, Giménez; Molina 2012).

\section{CONCLUSIONES:}

Se ha demostrado que los jugadores boyacenses poseen estatura y peso promedios, sin importar la posición de juego.

Se evidencia que poseen un componente muscular y graso adecuado para la competencia lo cual muestra características específicas por la ubicación geográfica.

El componente mesomórfico es predominante sobre el resto de los componentes del somatotipo, A través del presente estudio, realizado con jugadores de futbol sala del departamento de Boyacá, se ha podido demostrar un somatotipo global mesomórfico balanceado, (predominio muscular sobre el graso).

Es importante destacar que la composición corporal incidí directamente en la elección del módulo táctico defensivo y ofensivo.

\section{REFERENCIAS BIBLIOGRAFICAS:}

ALBA, A. (2005). Test funcionales. Kinesis.

ACERO J. (2002). Bases Biomecánicas para la Actividad Física Deportiva. Recuperado en: gse.com/antropometria-biomecanica-codificacionvertical-de-macro-indices-corpo

ACERO, J. (2002). Evaluación antropométrica y motriz condicional de niños y adolescentes. Recuperado en: https://books.google.com.co/books?isbn=95883 19110

DAZA, L. (2007). Evaluación clínica-funcional del movimiento corporal humano. Panamericana.

HEATH, CARTER (1967). Clasificación corporal. Recuperado en: https://sites.google.com/site/calculodelacomposi cioncorporal/home/somatotipo

GIMÉNEZ, C; MOLINA, L. (2012). Estudio Comparativo De La Composición Corporal Y EI Somatotipo Entre Jugadores De Fútbol Sala Universitario Y Profesional De La Región Suroccidente De Colombia. Univalle.

LENTINI, N; col. (2012). Estudio somatotipo en deportistas de alto rendimiento argentinos. Recuperado en: G-SE.com

MARROQUÍN, B; PRADO, A; DOMÍNGUEZ, G. (2008). Caracterización antropométrica, somatotipo y estado nutricional de los jugadores de fútbol sala categoría sub 17 masculino participantes en el torneo nacional, yumbo valle del cauca.

MOLINA, L.; JIMENEZ, C.; (2012). Estudio comparativo de la composición corporal y el somatotipo entre jugadores de fútbol sala universitario y profesional de la región suroccidente de Colombia. 
VALTUEÑA S, ARIJA V, SALAS J. (1996). Estado actual de los métodos de evaluación de la composición corporal: descripción, reproducibilidad, precisión, ámbitos de aplicación, seguridad, coste y perspectivas de futuro. Med Clin (Barcelona) 